\title{
Effects of Axonal Topology on the Somatic Modulation of Synaptic Outputs
}

\author{
Takuya Sasaki, Norio Matsuki, and Yuji Ikegaya \\ Laboratory of Chemical Pharmacology, Graduate School of Pharmaceutical Sciences, University of Tokyo, 7-3-1 Hongo, Bunkyo-ku, Tokyo 113-0033, Japan
}

\begin{abstract}
Depolarization of the neuronal soma augments synaptic output onto postsynaptic neurons via long-range, axonal cable properties. Here, we report that the range of this somatic influence is spatially restricted by not only axonal path length but also a branching-dependent decrease in axon diameter. Cell-attached recordings of action potentials (APs) from multiple axon branches of a rat hippocampal CA3 pyramidal cell revealed that an AP was broadened following a $20 \mathrm{mV}$ depolarization of the soma and reverted to a normal width during propagation down the axon. The narrowing of the AP depended on the distance traveled by the AP and on the number of axon branch points through which the AP passed. These findings were confirmed by optical imaging of AP-induced calcium elevations in presynaptic boutons, suggesting that the somatic membrane potential modifies synaptic outputs near the soma but not long-projection outputs. Consistent with this prediction, whole-cell recordings from synaptically connected neurons revealed that depolarization of presynaptic CA3 pyramidal cells facilitated synaptic transmission to nearby CA3 pyramidal cells, but not to distant pyramidal cells in CA3 or CA1. Therefore, axonal geometry enables the differential modulation of synaptic output depending on target location.
\end{abstract}

\section{Introduction}

Chemical neurotransmission is conventionally considered a local event that occurs at synaptic junctions. However, several pioneering studies have demonstrated previously that the membrane potential of the neuronal cell body before action potential (AP) generation affects neurotransmission at synapses that are distant from the cell body (Alle and Geiger, 2006, 2008; Shu et al., 2006; Kole et al., 2007; Scott et al., 2008; Christie et al., 2011). Although the underlying mechanisms remain controversial, the long-distance effect of the axon is mediated, at least in part, by an analog-like distortion of the AP waveform (Shu et al., 2006, 2007; Kole et al., 2007); APs originating from more depolarized membrane potentials are broader, and the broadened APs facilitate downstream synaptic transmission. This form of synaptic augmentation may be mediated by enhanced calcium dynamics at presynaptic terminals (Awatramani et al., 2005; Hori and Takahashi, 2009; Christie et al., 2011) (but see Alle and Geiger, 2008; Scott et al., 2008).

This somatic depolarization is reported to decay along the axon with length constants $(\lambda)$ of $430 \mu \mathrm{m}$ in rat hippocampal mossy fibers (Alle and Geiger, 2006), $417 \mu \mathrm{m}$ in layer 5 pyramidal cells in ferret prefrontal cortex (Shu et al., 2006), and $553 \mu \mathrm{m}$ of layer 5 pyramidal cells in rat somatosensory cortex (Kole et al., 2007). On the other hand, cable theory predicts that these $\lambda$ val-

\footnotetext{
Received 0ct. 24, 2011; revised Jan. 3, 2012; accepted Jan. 4, 2012.

Author contributions: N.M. and Y.I. designed research; T.S. performed research; T.S. analyzed data; T.S. and Y.I. wrote the paper.

This work was supported in part by Grants-in-Aid for Science Research 18021008, 22115003, 22115013, 22650080, and 22680025 from the Ministry of Education, Culture, Sports, Science and Technology of Japan; the Suzuken Memorial Foundation; the Kanae Foundation for the Promotion of Medical Science; the Daiichi-Sankyo Foundation of Life Science; and Funding Program for Next Generation World-Leading Researchers Grant LSO23.

Correspondence should be addressed to Yuji Ikegaya at the above address. E-mail: ikegaya@mol.f.u-tokyo.ac.jp. DOI:10.1523/JNEUROSCI.5365-11.2012

Copyright $\odot 2012$ the authors $\quad 0270-6474 / 12 / 322868-09 \$ 15.00 / 0$
}

ues are variable depending on cylinder diameter and the cable wiring pattern (Rall, 1959). Therefore, we hypothesized that AP modulation by somatic depolarization decays depending on not only axonal length but also branching. Cortical neurons often produce highly arborized axons; therefore, their somatic influence is expected to decrease rapidly during axon conduction and to differ markedly between proximal and distal synapses. However, this hypothesis has not been tested.

To test this hypothesis, we focused on hippocampal CA3 pyramidal cells because the axons in these neurons are locally arborized and synapse simultaneously on different postsynaptic targets, i.e., CA3 pyramidal cells (proximal or distal target) and CA1 pyramidal cells (distal target). In acute slice preparations, most Schaffer collaterals (long-projecting axons from CA3 to CA1) are severed, making it difficult to locate synaptically connected CA3-to-CA1 pairs. Therefore, we chose to use hippocampal slice cultures. The cultured network self-restores to an in vivo-like connectivity (Takahashi et al., 2010), providing a unique opportunity for direct comparisons between proximal and distal axonal connections in the same preparation, even in the same presynaptic neuron. Moreover, we previously established three imaging techniques that can be used to address our hypothesis: (1) fluorescent patch-clamp pipettes that enable direct recording from intact axon branches (Ishikawa et al., 2010; Sasaki et al., 2011; Takahashi et al., 2012), (2) wide-field confocal imaging that simultaneously monitors the calcium activity of presynaptic varicosities from multiple axon branches (Sasaki et al., 2011), and (3) reversal optical trawling (ROTing), which is a high-throughput, large-scale synapse mapping method that identifies long-distance synaptic connections (Sasaki et al., 2009; Takahashi et al., 2010). Using these techniques, we demonstrate that axonal topology influences the somatic modulation of APs and subsequent synaptic output. 


\section{Materials and Methods}

Animal experiment ethics. All experiments were performed with the approval of the animal experiment ethics committee at the University of Tokyo (approval number 19-43) and according to the University of Tokyo guidelines for the care and use of laboratory animals.

Hippocampal slice culture preparations. Postnatal day 7 Wistar/ST rats of either sex were anesthetized with ketamine/xylazine and chilled on ice. The brains were removed and cut horizontally into $300-\mu \mathrm{m}$-thick slices using a DTK-1500 vibratome (Dosaka). During the generation of slices, brain tissues were submerged in aerated, ice-cold Gey's balanced salt solution supplemented with $25 \mathrm{~mm}$ glucose. Entorhinohippocampal slices were cultivated for 7-14 d on Omnipore membrane filters (JHWP02500; $\varphi 25$ mm; Millipore) (Koyama et al., 2007). Cultures were incubated in $1 \mathrm{ml}$ of $50 \%$ minimal essential medium, 25\% HBSS (Invitrogen), and $25 \%$ horse serum (Cell Culture Laboratory) in a humidified incubator at $37^{\circ} \mathrm{C}$ in $5 \% \mathrm{CO}_{2}$. The medium was changed every $3.5 \mathrm{~d}$. Cultured slices were analyzed after $8-11 \mathrm{~d}$ in vitro unless specified otherwise.

Patch-clamp recordings. A cultured slice was placed in a recording chamber and perfused at 3-4 $\mathrm{ml} / \mathrm{min}$ with artificial CSF (ACSF) containing the following (in $\mathrm{mm}$ ) unless specified otherwise: $127 \mathrm{NaCl}, 26$ $\mathrm{NaHCO}_{3}, 3.3 \mathrm{KCl}, 1.24 \mathrm{KH}_{2} \mathrm{PO}_{4}, 3.0 \mathrm{MgSO}_{4}, 3.0 \mathrm{CaCl}_{2}$, and 10 glucose at $30-32^{\circ} \mathrm{C}$. Whole-cell recordings were performed from visually identified pyramidal cells. Patch pipettes (5-7 M $\Omega$ ) were filled with $135 \mathrm{~mm}$ K-gluconate, $4 \mathrm{~mm} \mathrm{KCl}, 10 \mathrm{~mm}$ HEPES, $10 \mathrm{~mm}$ phosphocreatine, $4 \mathrm{~mm}$ MgATP, 0.3 mм NaGTP, and $200 \mu \mathrm{m}$ Alexa Fluor 488 hydrazide, pH 7.2 (Invitrogen). Axonal cell-attached recordings were obtained using fluorophore-coated pipettes (Ishikawa et al., 2010). Immediately before use, the glass pipettes $(9-12 \mathrm{M} \Omega$ ) were filled with ACSF, and the tips were immersed for $5-10 \mathrm{~s}$ in $0.02 \%$ bovine serum albumin Alexa Fluor 488 conjugate (A-13100; Invitrogen). Action potentials were evoked by brief current injection (2-3 ms; $1-2 \mathrm{nA}$ ) into the soma. Signals were recorded at $20-100 \mathrm{kHz}$ using two Multiclamp 700B amplifiers (Molecular Devices). Fast and slow pipette capacitive transients were minimized in the cell-attached configuration. No compensation was made to cancel access resistance. To apply the electrical stimulation, glass pipettes were filled with ACSF and placed in the dentate hilus. Single-pulse stimuli ( $80 \mu \mathrm{s}$; $100-200 \mu \mathrm{A}$ ) were applied at intervals of $>10 \mathrm{~s}$.

Presynaptic calcium imaging. CA3 pyramidal cells were whole-cell patched using a pipette loaded with $250 \mu \mathrm{M}$ Oregon Green 488 BAPTA-1 (OGB1) potassium salt. After allowing 30-60 min for intra-axonal dye diffusion, axon branches were monitored at 50 frames per second (fps) using a Nipkow-disk confocal unit (CSU-X1; Yokogawa Electric), a back-illuminated CCD camera (iXon DU897; Andor), and a waterimmersion objective lens (40X; NA, 0.80; Nikon). Fluorophores were excited at $488 \mathrm{~nm}$ with a laser diode (HPU50101PFS; FITEL) and visualized using a $507 \mathrm{~nm}$ long-pass emission filter. The AP-induced calcium increase was measured as the mean change in the fluorescence intensity of five neighboring synaptic varicosities (ROI, $2 \times 3 \mu \mathrm{m}^{2}$ each) averaged for five trials to enhance the signal-to-noise ratio.

ROTing. ROTing was used to detect synaptically coupled neurons ( $\mathrm{Sa}-$ saki et al., 2009). For dye loading, a glass pipette (1-3 M $\Omega$ ) was filled with ACSF containing $200 \mu \mathrm{M}$ OGB1 AM (Invitrogen) and inserted into the CA3 pyramidal cell layer. Using intrapipette pressure, neurons were bolus loaded with the calcium indicator. One or two CA3 or CA1 pyramidal cells were voltage clamped at $-70 \mathrm{mV}$, and $10 \mu \mathrm{M}$ glutamate was applied locally through iontophoretic pipettes $(\sim 1 \mathrm{M} \Omega, 3-10 \mu \mathrm{A}$ for $1-5 \mathrm{~s}$ each). This low concentration of glutamate evoked APs in a few CA3 neurons. The iontophoretic pipette was slowly moved over the CA3 networks, and the evoked APs were monitored at $50 \mathrm{fps}$ using Nipkow-disk confocal microscopy with a water-immersion objective lens (16×; NA, 0.80 ; Nikon). APs were determined based on the onset times of individual calcium transients using an automatic machine-learning algorithm (Sasaki et al., 2008). To determine which pyramidal neurons were candidate presynaptic targets for subsequent patch clamping, the AP timing was compared to the onset of EPSCs recorded in the patch-clamped neurons.

In silico experiments. Simulation was conducted in the Visual Basic environment using the conventional Hodgkin-Huxley $(\mathrm{HH})$ model, which included potassium leak conductance, voltage-sensitive sodium channel conductance, and delayed-rectifier potassium channel conductance as follows (Hodgkin and Huxley, 1952; Dayan and Abbott, 2001):

$$
C_{\mathrm{m}} \frac{d V}{d t}=g_{\mathrm{Na}}\left(V-E_{\mathrm{Na}}\right)+g_{\mathrm{K}}\left(V-E_{\mathrm{K}}\right)+\bar{g}_{\mathrm{L}}\left(V-E_{\mathrm{L}}\right)+g_{\text {syn }}\left(V-E_{\text {syn }}\right),
$$

where $C_{\mathrm{m}}=1 \mu \mathrm{F} / \mathrm{cm}^{2}$ is the specific membrane capacity; $V$ is the difference between the resting potential and the instantaneous membrane potential; $E_{\mathrm{Na}}=115 \mathrm{mV}, E_{\mathrm{K}}=-12 \mathrm{mV}, E_{\mathrm{L}}=10.6 \mathrm{mV}$, and $E_{\text {syn }}=70 \mathrm{mV}$ are the reversal potentials for the sodium, potassium, leakage, and synaptic current components, respectively; $g_{\mathrm{Na}}$ and $g_{\mathrm{K}}$ are the $V$-dependent ionic conductance through the sodium and potassium components, respectively; $\bar{g}_{\mathrm{L}}=0.3 \mathrm{mS} / \mathrm{cm}^{2}$ is the leakage current component; and $g_{\text {syn }}$ is the synaptic conductance used to evoke an action potential. $g_{\text {syn }}$ was changed from 0 to $0.2 \mathrm{mS} / \mathrm{cm}^{2}$ to generate an AP in the HH neuron. $g_{\mathrm{Na}}$ and $g_{\mathrm{K}}$ are given as follows:

$$
\begin{gathered}
g_{\mathrm{Na}}=\bar{g}_{\mathrm{Na}} m^{3} h, \\
g_{\mathrm{K}}=\bar{g}_{\mathrm{K}} n^{4} f,
\end{gathered}
$$

where $\bar{g}_{\mathrm{Na}}=120 \mathrm{mS} / \mathrm{cm}^{2}$ and $\bar{g}_{\mathrm{K}}=36 \mathrm{mS} / \mathrm{cm}^{2}$ are the maximal ionic conductances, and $m, n$, and $h$ are dynamic activation (or gating) variables. They evolve according to the following differential equations (Dayan and Abbott, 2001):

$$
\begin{aligned}
& \frac{d m}{d t}=\alpha_{m}(V)(1-m)-\beta_{m}(V) m ; \\
& \frac{d h}{d t}=\alpha_{h}(V)(1-h)-\beta_{h}(V) h ; \\
& \frac{d n}{d t}=\alpha_{n}(V)(1-n)-\beta_{n}(V) n .
\end{aligned}
$$

The various functions $\alpha$ and $\beta$ are empirical functions of $V$ and given as follows (Dayan and Abbott, 2001):

$$
\begin{gathered}
\alpha_{m}=(2.5-0.1 V) /\left(e^{(2.5-0.1 V)}-1\right) ; \\
\beta_{m}=4 e^{-V / 18} ; \\
\alpha_{h}=0.07 e^{-V / 20} ; \\
\beta_{h}=1 /\left(e^{(3-0.1 V)}+1\right) ; \\
\alpha_{n}=(0.1-0.01 V) /\left(e^{(1-0.1 V)}-1\right) ; \\
\beta_{n}=0.125 e^{-V / 80} .
\end{gathered}
$$

$f$ in Equation 3 is the inactivation function of voltage-dependent potassium channels and is defined as $f=1 /\left(1+0.15 e^{0.1\left(V_{\text {init }}+20\right)}\right)$, where $V_{\text {init }}$, ranging from 0 to $40 \mathrm{mV}$, is the difference between the resting potential and the potential at the time when $g_{\text {syn }}$ was changed to 0.2 $\mathrm{mS} / \mathrm{cm}^{2}$. Simulations were conducted at a step size of $20 \mu \mathrm{s}$.

Data are reported as the mean \pm SE.

\section{Results}

\section{Computational prediction of somatic AP modulation}

Whole-cell recordings were obtained from hippocampal CA3 pyramidal cells. To examine how the membrane potential immediately before AP initiation affects the subsequent AP waveform, a small step-pulse current was injected into the cell body to depolarize presynaptic neurons by $5-35 \mathrm{mV}$ for $1 \mathrm{~s}$ before AP firing. The initiation time of an AP was determined at a threshold of $d^{2} V / d t^{2}=0.25$ $\mu \mathrm{V} / \mu \mathrm{s}^{2}$, and the AP width was defined as its half-maximum width, i.e., the duration at $50 \%$ of the voltage difference between AP initiation and AP peak. Somatic depolarization in- 
creased AP width (Fig. 1A, left), and larger depolarizations produced more broadened APs (Fig. 1B). This relationship was supralinear and apparently approximated by $-7.5 \times\left(1-e^{-0.06 \Delta V_{\text {soma }}}\right)$, where $\Delta V_{\text {soma }}$ is the voltage difference from the resting potential of the soma. These experimental results were replicated using the conventional $\mathrm{HH}$ model (Fig. $1 A$, right, $B$ ).

Using the assumption that the axon cable is a semi-infinite homogeneous cylindrical compartment, cable theory predicts that the voltage difference induced in the soma decays along the longitudinal axis of the axon as $\Delta V(x)=\Delta V_{\text {soma }} e^{-x / \lambda}$, where $x$ is the path length from the soma, and $\lambda$ is the length constant (Dayan and Abbott, 2001). Because of the nature of the supralinear relationship between $\Delta V$ and AP broadening (Fig. $1 \mathrm{~B}$ ), the length constant in the recovery of AP broadening during axonal conduction $\left(\lambda_{\mathrm{AP}}\right)$ is smaller than $\lambda$. For example, the $\mathrm{HH}$ simulation indicated that $\lambda_{\mathrm{AP}}$ was $0.72 \times \lambda$ when $\Delta V_{\text {soma }}=20 \mathrm{mV}$ (Fig. 1C). $\lambda_{\mathrm{AP}}$ depended on $\Delta V_{\text {soma }}$ and was consistently smaller than $\lambda$ (Fig. $1 D$ ), indicating that the AP-broadening effect of $\Delta V_{\text {soma }}$ is less than that expected from the linear prediction of simple cable properties. In other words, $\Delta V_{\text {soma }}$-induced AP broadening recovers more rapidly during axonal conduction than simply expected by the axon length constant.

The above simulations may be oversimplified because real axonal tubes are inhomogeneous and highly branched. One of the most influential factors is the axon diameter $(d)$. According to cable theory, $\lambda$ is proportional to the square root of $d$ (Fig. 2A, left) (Dayan and Abbott, 2001). Given that the sibling axon branches are usually narrower than the parent axon, $\lambda$ may vary between prebranch and postbranch cables (Fig. $2 \mathrm{~A}$, right) (Rall, 1959; Dayan and Abbott, 2001).

To estimate the impact of axonal branching on $\lambda$, we reconstructed the axons of CA3 pyramidal cells that were intracellularly labeled with the membrane-impermeable fluorescent dye Alexa Fluor 488 hydrazide through patch-clamp pipettes (Fig. $2 B$ ). The dendrogram of the axonal branch patterns (Fig. $2 C$ ) indicates that the number of branch points between the soma and a given segment on the axon increased with the path length between the soma and the analyzed segment. These branching patterns were similar to those observed in the hippocampus in vivo (Li et al., 1994; Wittner et al., 2007).

The axon diameter cannot be measured directly by optical microscopy because it is often $<1 \mu \mathrm{m}$ and smaller than the point spread function (Conchello and Lichtman, 2005). Therefore, by assuming that the total fluorescence intensity $(F)$ in a given axonal segment is proportional to the axonal volume [i.e., $F \propto$ volume $=\pi(d / 2)^{2} \times$ unit length], we estimated the relative axon diameter as the square root of the Alexa Fluor 488 fluorescence intensity, i.e., $d \propto F^{1 / 2}$. In neurons held in the whole-cell configuration for $>60 \mathrm{~min}$, which was long enough to reach the steady state of dye diffusion in the axon, the $F$ values were measured from three $5 \mu \mathrm{m}$ sub-branch segments (i.e., a parent and two siblings) within $10 \mu \mathrm{m}$ of each branch point, and the background autofluorescence $F_{\text {back }}$ was measured from the parenchymal re- gion $10 \mu \mathrm{m}$ apart from to the branch point and subtracted from $F$. In most branch points, the putative diameters $\left(F-F_{\text {back }}\right)^{1 / 2}$ of sibling axonal branches were smaller than the parent axonal diameter (Fig. 2D, black dots). According to this plot, branchdependent decreases in the axon diameters were particularly marked within a distance of $200 \mu \mathrm{m}$ from the soma. As control experiments, we measured $F$ from two axonal points $(20 \mu \mathrm{m}$ apart) without a branch point and found no apparent change in the intensity between such a short interval; the diameter ratio was $0.99 \pm 0.07$ (Fig. $2 D$, purple line, shadow) $(n=108$ randomly selected axonal shafts).

In the dendrites of some types of neurons, the fiber diameters are known to conform to the so-called 3/2 power law (Rall, 1959; Turner and Schwartzkroin, 1980; Desmond and Levy, 1984), in which the $3 / 2$ power of the parent segment diameter $d_{1}$ is equal to the sum of the two sibling segment diameters $d_{2}$ and $d_{3}$, both raised to the $3 / 2$ power, i.e., $d_{1}^{3 / 2}=d_{2}^{3 / 2}+d_{3}^{3 / 2}$. Note that if this relationship holds between any parent dendrite and its siblings, the complex dendritic tree of the neuron becomes equivalent to an unbranched cylinder for simplified electronic calculations. Surprisingly, the $3 / 2$ power relationship has not been addressed in axonal branching. We found that the axon diameters show a complicated relationship (Fig. 2E). At branches close to the soma, the $d_{1}^{3 / 2}$ value tended to be smaller than $d_{2}^{3 / 2}+d_{3}^{3 / 2}$, whereas it was larger than $d_{2}^{3 / 2}+d_{3}^{3 / 2}$ at distant branches. These results raise the possibility that $\Delta V_{\text {soma }}$-induced AP broadening recovers more rapidly in branched axons, particularly in the vicinity of the soma, than in an unbranched axonal cylinder.

Using these detailed morphological data, we simulated the $\mathrm{HH}$ dynamics of the AP width during axonal conduction when the soma of the neuron reconstructed in Figure $2 B$ was depolarized by $\Delta V_{\text {soma }}=20 \mathrm{mV}$. $\Delta V$ was decayed along the axon length by an simple exponential decay equation, based on two assump- 

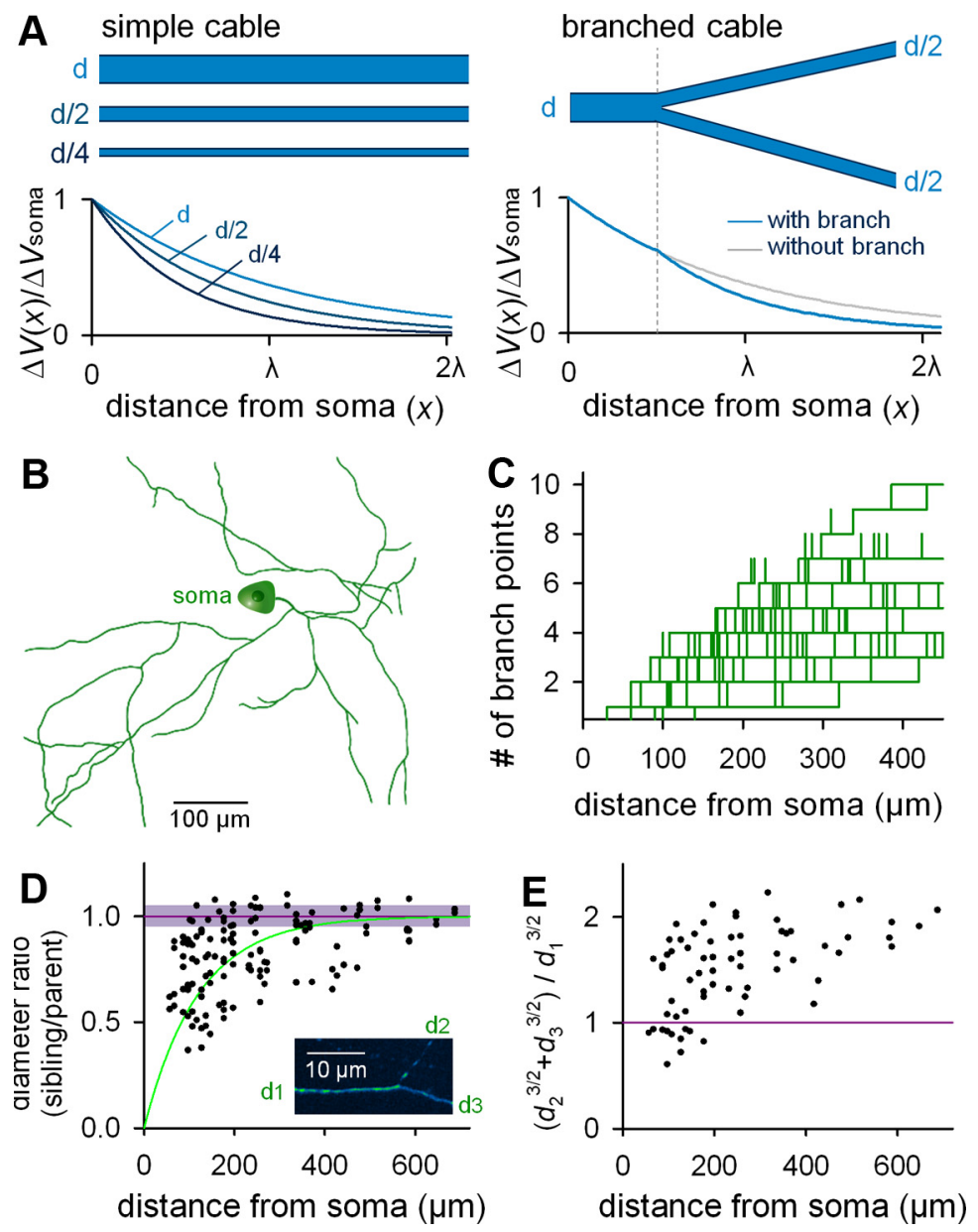

Figure 2. Anatomical characterization of the axonal arborization of CA3 pyramidal cells ex vivo. $\boldsymbol{A}$, Cable theory predicts that recovery of the depolarized membrane potential $(\Delta V)$ along the longitudinal axis of the cable depends on cable diameter (left) and branching (right). $\boldsymbol{B}$, Representative reconstruction of the CA3 pyramidal cell axon. $\boldsymbol{C}$, The number of branch points between the soma and a given point on the axon is plotted as a function of the path length from the soma to the examined point ( $n=5$ cells). $D$, The ratio of the diameter of a pre-branch "parent" axon segment $\left(d_{1}\right)$ to those of the two postbranch "sibling " axon segments $\left(d_{2}\right.$ or $\left.d_{3}\right)$ is plotted as a function of the path length from the soma. The square root of the Alexa Fluor 488 fluorescence intensity of an axon segment within $10 \mu \mathrm{m}$ of a given branch point was used as an estimate of axon diameter ( $n=5$ cells). As control, the mean and SD of the ratio in the fluorescence intensities between two axon points at a path interval of $20 \mu \mathrm{m}$ without branching are shown in the purple line and shade. Inset, Example of high-magnification of a branching point. $\boldsymbol{E}$, Ratios of the $3 / 2$ power values of axon diameters [i.e., $\left(d_{2}^{3 / 2}+d_{3}^{3 / 2}\right) / d_{1}^{3 / 2}$ ] are plotted as a function of the path length from the soma $(n=5$ cells $)$. $\boldsymbol{F}$, Computational simulation of depolarization-induced AP width along arborized axons when the axonal length constant $(\lambda)$ is constant throughout the axon (left, without branch decay) or when a branch-dependent decrease in the axonal diameters was considered (right, with branch decay). Simulation was conducted on the neuron reconstructed in $\boldsymbol{B}$.

tions (1) $\lambda$ changes at every branch point, depending on a decrease in $d\left(\lambda \propto d^{1 / 2}\right)$, and (2) $d$ is constant in a axon shaft between two successive branch points. $\lambda$ at the axon initial segment was set to be $450 \mu \mathrm{m}$. The AP waveform was determined by $\Delta V$ on the spot. We found that $\Delta V_{\text {soma }}$-induced changes in the AP width occurred in a shorter range (Fig. $3 A$, right) compared to those when the branch effect was not considered (i.e., when $\Delta V$ was assumed to decay depending simply on the axon path length) (Fig. 3A, left). To determine how far somatic AP modulation is maintained along the axon, 80 axonal segments were randomly selected from the axon arbor and plotted against the axon path length (Fig. $3 B$ ) and the number of upstream branch points (Fig. $3 C$ ). We first fitted the data of Figure $3 B$ to an exponential decay function using the least-squares method. The length constants $\lambda_{\mathrm{AP}}$ was $344 \mu \mathrm{m}$ without branch decay (Fig. $3 B$, left; $F_{(1,78)}=\infty$; $p=0.00$ ) and $172 \mu \mathrm{m}$ with branch decay (Fig. $3 B$, right; $F_{(1,78)}=$ 1244.9; $p<0.001)$, indicating that depolarization-broadened
APs reverted more rapidly when the branching effect was taken into consideration. Because of the interdependence between the distance and the number of branch points (Fig. 2C), this AP recovery can also be considered to depend on the number of branches over which an AP passes; that is, increased narrowing of the widened APs occur at axonal branch points. We thus fitted the data of Figure $3 C$ to the exponential decay function $e^{-n / \lambda_{A P}}$, where $n$ is the number of upstream branches at the focused point. $\lambda_{\mathrm{AP}}$ was 4.7 branches without branch decay (Fig. $3 C$, left; $\left.F_{(1,78)}=10.7 ; p<0.001\right)$ and 2.1 branches with branch decay (Fig. 3C, right; $\left.F_{(1,78)}=294.7 ; p<0.001\right)$, indicating again that the broadened APs reverted more rapidly when the branching effect was considered.

Together, our simulations suggest that the axon length constant $\lambda$ overestimates the spatial range of the $\Delta V_{\text {soma }}$ influence on the AP waveform $\left(\lambda_{\mathrm{AP}}\right)$ because the AP width is nonlinearly related to $\Delta V$ and because $\lambda$ decreases at branch points. We expect that this spatial restriction is particularly prominent in axons that are highly arborized in the vicinity of the axon initial segments, such as associational fibers of CA3 pyramidal cells (Li et al., 1994; Wittner et al., 2007).

\section{Extracellular APs monitored by cell-attached recording}

We experimentally examined the effect of $\Delta V_{\text {soma }}$ on the AP waveform during conduction of branched axons by performing cell-attached recordings of intact axons. Alexa Fluor 488 hydrazide was injected into CA3 pyramidal cells through whole-cell patch-clamp pipettes, and visualized axons were targeted using fluorophore-coated pipettes under real-time confocal control (Fig. $4 A$ ). APs were evoked by brief current injections into the soma and extracellularly recorded at the axon as sharp sink potentials (eAPs). The halfmaximum width of eAPs was increased by a $20 \mathrm{mV}$ depolarization of the cell body before AP firing (Fig. $4 B$ ).

Although eAPs recorded from local field potentials (LFPs) are thought to reflect the derivative of intracellularly recorded APs (iAPs), the origin of eAPs monitored by cell-attached recording is controversial (Raastad and Shepherd, 2003; Khaliq and Raman, 2006; Perkins, 2006). Therefore, we simultaneously recorded and compared the waveforms of iAPs (whole cell) and eAPs (cell attached) from cell bodies of CA3 pyramidal cells and eAPs recorded in LFPs (Fig. 4C). These three traces were phase-space analyzed (Fig. $4 D$ ). Although the eAPs (cell attached) were not exactly the inverse or derivative of the iAPs, they were similar to the inverted waveform of the iAPs. This was in contrast to the eAPs in LFPs, which were similar to the inverted waveform of the first derivative of the iAPs.

We then compared the sensitivity of the iAP and eAP waveforms to $20 \mathrm{mV}$ depolarization; $10 \mathrm{nM}$ tetrodotoxin, a voltage- 

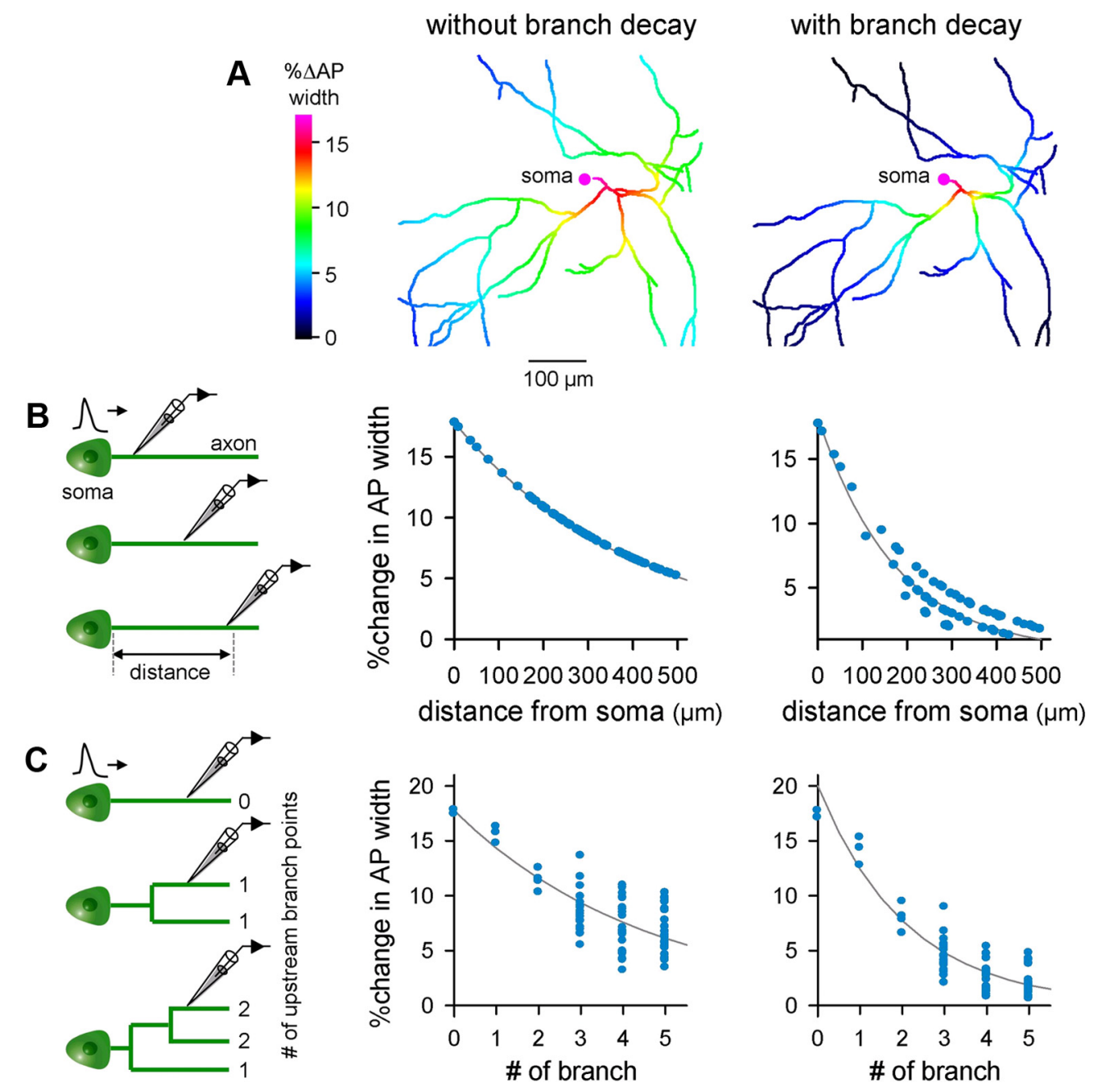

Figure 3. HH simulation of depolarization-induced AP width along arborized axons. $A$, Simulation was conducted on the neuron reconstructed in Figure $2 B$. Left, The axonal length constant $(\lambda=$ $450 \mu \mathrm{m}$ ) is constant throughout the axon (without branch decay). Right, A branch-dependent decrease in the axonal diameters was considered (with branch decay). $\boldsymbol{B}$, Distance-dependent recovery of depolarization-broadened APs. The curve indicates the least-square best fit of the exponential decay function (left, without branch decay; $\% \Delta$ width $=17.9 \times e^{-d / 344} ; d$, distance from the soma; $F_{(1,78)}=\infty ; p=0.0 ;$ right, with branch decay; $\% \Delta$ width $\left.=18.3 \times e^{-d / 172} ; F_{(1,78)}=1244.9 ; p<0.001\right)$. C, Branch number-dependent recovery of depolarization-broadened APs. The curve indicates the best fit of the exponential decay function (left, $\% \Delta$ width $=17.7 \times e^{-n / 4.7} ; n$, number of upstream branches; $F_{(1,78)}=10.7 ; p<0.001 ;$ right; $\% \Delta$ width $=20.0 \times e^{-n / 2.1}$; $\left.F_{(1,78)}=294.7 ; p<0.001\right)$. Data presented here were collected from 14 recordings.

sensitive sodium channel inhibitor; and $10 \mu \mathrm{M}$ 4-aminopyridine (4-AP), a putative inhibitor of the Kv1 family of voltage-activated potassium channels. The AP amplitude, 20 to $80 \%$ rise time, decay time constant, and half-width were used to evaluate AP modulation (Fig. $4 E$ ). In both iAPs and eAPs, tetrodotoxin altered all of the AP waveform parameters measured, whereas depolarization and 4-AP primarily affected the AP decay and width, i.e., the late phase of the AP. Importantly, as expected from the data in Figure $4 D$, the changes in the AP width were almost linearly proportional to the changes in the iAP width (Fig. $4 F ; r=$ $0.58 ; p=0.03 ; n=12$ ). Accordingly, the eAP width can serve as an indicator of $\mathrm{iAP}$ width.

\section{Effects of axonal length and branching on AP modulation}

To determine how far somatic AP modulation is maintained along the axon, we performed dual or single recordings of eAPs from different axonal points (Fig. $5 A$ ). Broadened APs gradually reverted to a normal width as a function of the axonal distance over which they traveled (Fig. 5B). This curve was fitted to an exponential decay function using the least-squares method. The decay constant was $145 \mu \mathrm{m}\left(F_{(1,12)}=229.8 ; p=0.002\right)$. Addi- tionally, we found that increased narrowing of the widened APs also occurred at axonal branch points, with a decay constant of 2.9 branches (Fig. $5 C ; F_{(1,12)}=158.4 ; p=0.006$ ). These decay constants are more similar to the result of our $\mathrm{HH}$ simulation with branch decay than that without branch decay (Fig. $2 A$ ).

To investigate whether AP modulation influences the function of presynaptic terminals, we performed functional optical imaging. We used Nipkow disk confocal microscopy to measure intracellular calcium dynamics in axonal varicosities loaded anterogradely with OGB1 through somatic patch-clamp pipettes (Fig. 6A,B). This technique enables high-speed and wide-field monitoring without apparent phototoxicity (Sasaki et al., 2007). To enhance the signal-to-noise ratio, we averaged AP-evoked fluorescence signals from five neighboring synaptic varicosities. This measurement was further averaged across 6-10 successive trials, each of which alternated between the resting and $20 \mathrm{mV}$ depolarized states. After this spatiotemporal averaging, we could clearly observe depolarization-induced enhancement of APtriggered calcium increases in synaptic boutons, an effect that may be mediated by specific types of voltage-sensitive calcium channels (Yu et al., 2010; Christie et al., 2011). Data collected 


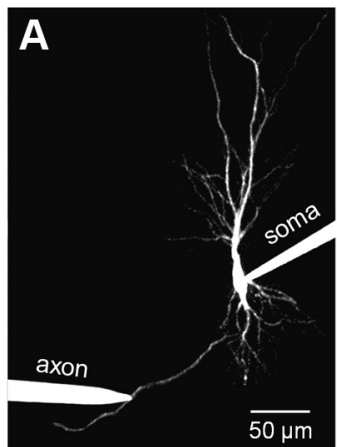

B

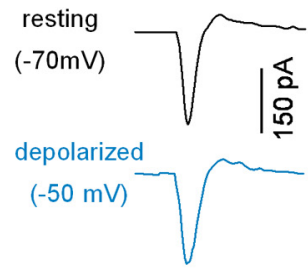

merged
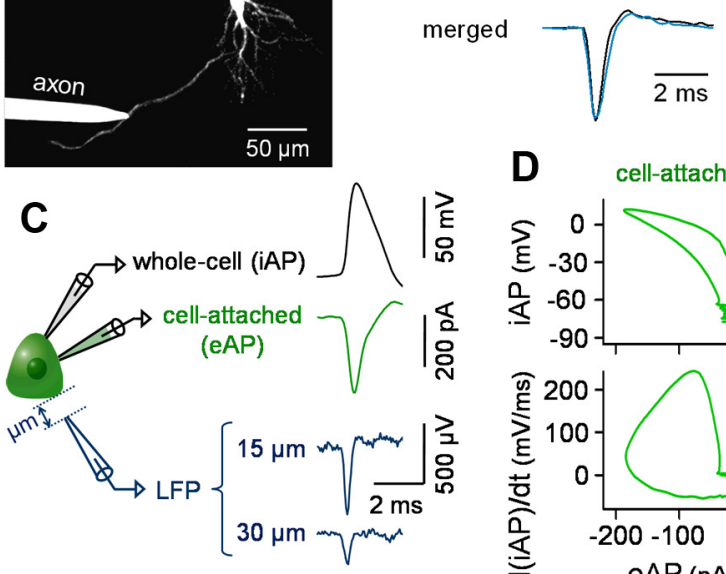

D cell-attached
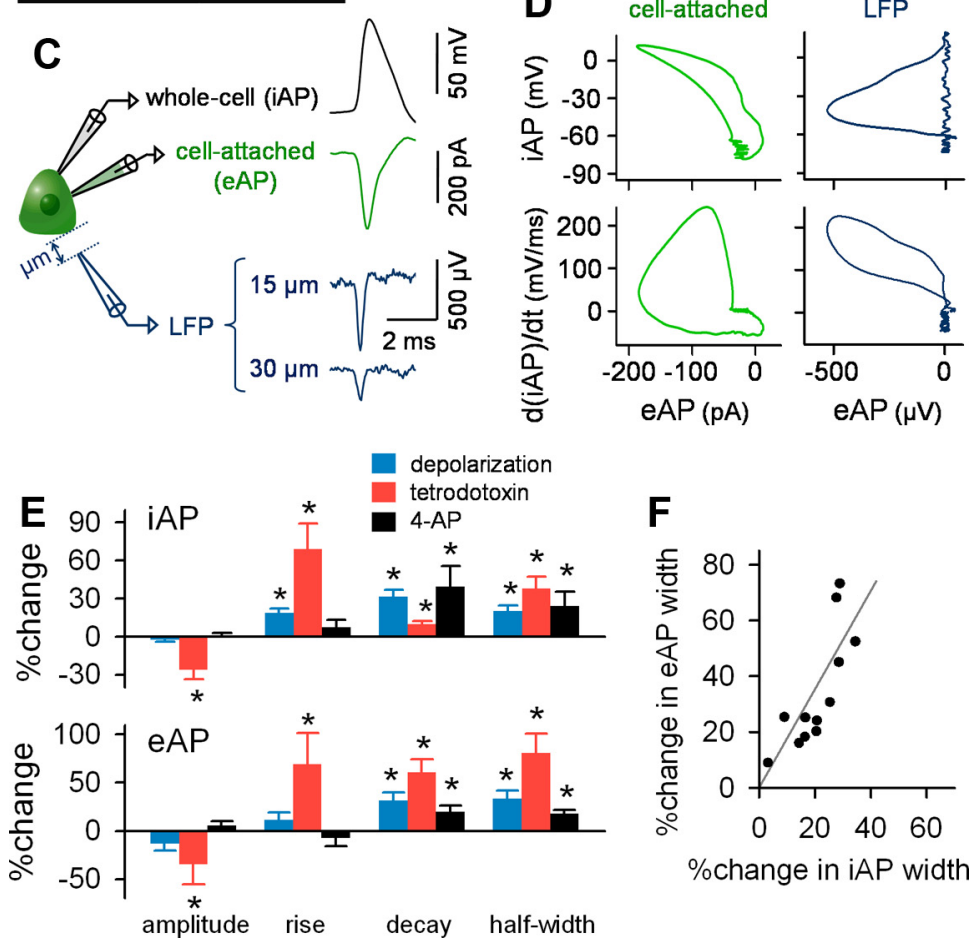

Figure 4. Cell-attached recording of eAPs recapitulates the features of iAPs. $\boldsymbol{A}$, Visually guided patch-clamp recording from the axon of a CA3 pyramidal cell. For visualization, the fluorescence contrast was emphasized around the axon tract. $\boldsymbol{B}$, Typical traces of eAPs recorded from the axon of a resting (black) or $20 \mathrm{mV}$-depolarized CA3 neuron (blue). $C$, Simultaneous triple recordings of iAP, eAP, and eAP from the soma of the same cell. LFPs were recorded 15 and $30 \mu \mathrm{m}$ from the cell body surface. $D$, Time-dependent evolution of the waveform of an eAP (left, cell attached; right, LFP at $15 \mu \mathrm{m}$ ) against the corresponding iAP waveform (top) and its derivative (bottom). $\boldsymbol{E}$, Effects of $20 \mathrm{mV}$ depolarization $(n=6$ cells), $10 \mathrm{~nm}$ tetrodotoxin $(n=4)$, and $10 \mu \mathrm{m} 4-\mathrm{AP}(n=4)$ on four AP-relevant parameters (i.e., amplitude, 20 to $80 \%$ rise time, decay constant, and amplitude half-width). ${ }^{*} p<0.01$, paired $t$ test. $\boldsymbol{F}$, Changes in eAP width are linearly proportional to changes in iAP width $(r=0.58 ; p=0.03)$.

from 81 axonal segments from seven cells were plotted against the distance the AP traveled or the axonal branch number (Fig. 6C). The depolarization-enhanced signal amplitude could be fitted to a single exponential decay function with a decay constant of either $176 \mu \mathrm{m}\left(F_{(1,79)}=30.2 ; p<0.001\right)$ or 3.4 branches $\left(F_{(1,79)}=\right.$ $11.2 ; p=0.0012)$.

Because we used relatively higher concentrations of divalent ions in the ACSF and a lower temperature to obtain these data, we repeated the same experiments under more physiological conditions $\left(3.5 \mathrm{~mm} \mathrm{~K}^{+}, 2.0 \mathrm{mM} \mathrm{Mg}^{2+}\right.$, and $1.8 \mathrm{mM} \mathrm{Ca}^{2+}$ at $\left.36^{\circ} \mathrm{C}\right)$. The best fit was given at $\tau_{x}=161 \mu \mathrm{m}$ and $\tau_{n}=3.1$ branches (single exponential fitting). We also used more mature slice preparations under our routine ionic compositions and temperature $(4.54 \mathrm{mM}$ $\mathrm{K}^{+}, 3.0 \mathrm{mM} \mathrm{Mg}^{2+}$, and $3.0 \mathrm{mM} \mathrm{Ca}^{2+}$ at $\left.30-32^{\circ} \mathrm{C}\right)$. After $20-22 \mathrm{~d}$ in vitro, the $\tau_{x}$ and $\tau_{n}$ values were found to be $159 \mu \mathrm{m}$ and 3.2 branches. In both conditions, $\tau_{x}$ and $\tau_{n}$ values tended to be smaller than that observed under our experimental conditions, although the difference was not statistically significant $(p>0.1 ; z$ test for two regression coefficients).

These decay constants for axon lengths and branch points were consistently similar to those obtained when branchingdependent decrease in the axon diameter was assumed in the $\mathrm{HH}$ model. However, branch point numbers and axonal path lengths are interdependent in real neurons (Fig. 2C), and one cannot strictly discriminate the independent effects of these two factors using the curve fitting method alone. Therefore, to examine whether branching actually impacts the AP decay, we compared the effect of $\Delta V_{\text {soma }}$ at two axonal segments that had an identical distance from the soma but different branch numbers from the soma. For this purpose, we chose calcium-imaging experiments, rather than axon patch-clamp recordings, because of higher experimental throughputs. In an example in Figure $6 D$ (top), calcium traces were recorded at a path distance of $209 \mu \mathrm{m}$ from the soma; the left and right traces were obtained from axon segments at which two and four branch points existed in the path from the soma, respectively. In the segment with two branches, the size of calcium transients increased to $122 \%$ in response to $\Delta V_{\text {soma }}$, whereas the four-branch segment did not show a change (95\%). Thus, this twobranch difference produced a $77.9 \%$ (95 of 122) drop of the $\Delta V_{\text {soma }}$ effect. We repeated similar data sampling from 28 pairs of the axonal segments that had differences of one or two branches and plotted the ratios of $\Delta V_{\text {soma }}$-induced increases in calcium transient sizes (Fig. 6D, bottom; $n=13$ cells). We found that even at the same distance, axonal segments with more branches produced significantly smaller modulations by $\Delta V_{\text {soma }}$; the mean ratio was $93.5 \pm 1.3 \%\left(n=28\right.$ pairs; $t_{(27)}$ $=5.14 ; p=2.0 \times 10^{-5}$, paired $t$ test $)$. These data are consistent with our theoretical hypothesis that somatic AP modulation is affected by axon length as well as branching.

Short-range somatic influence on CA3 synaptic transmission To examine the influence of somatic depolarization on synaptic transmission, we performed simultaneous recordings from synaptically connected pairs or triplets of CA3 and CA 1 pyramidal cells that were identified using the ROTing technique (Sasaki et al., 2009). For CA3 pairs, neurons located within $100 \mu \mathrm{m}$ of one another (close pair) or $>300 \mu \mathrm{m}$ apart (distant pair) were selected. Presynaptic CA3 neurons were current injected 50 times at 10 s intervals to evoke single action potentials, and unitary EPSCs (uEPSCs) were recorded from the postsynaptic cells (Fig. 7A).

The synaptic efficacy (the mean size of uEPSCs) of close CA3-toCA3, distant CA3-to-CA3, and CA3-to-CA1 transmission was compared between resting and $20 \mathrm{mV}$ depolarized states (Fig. 

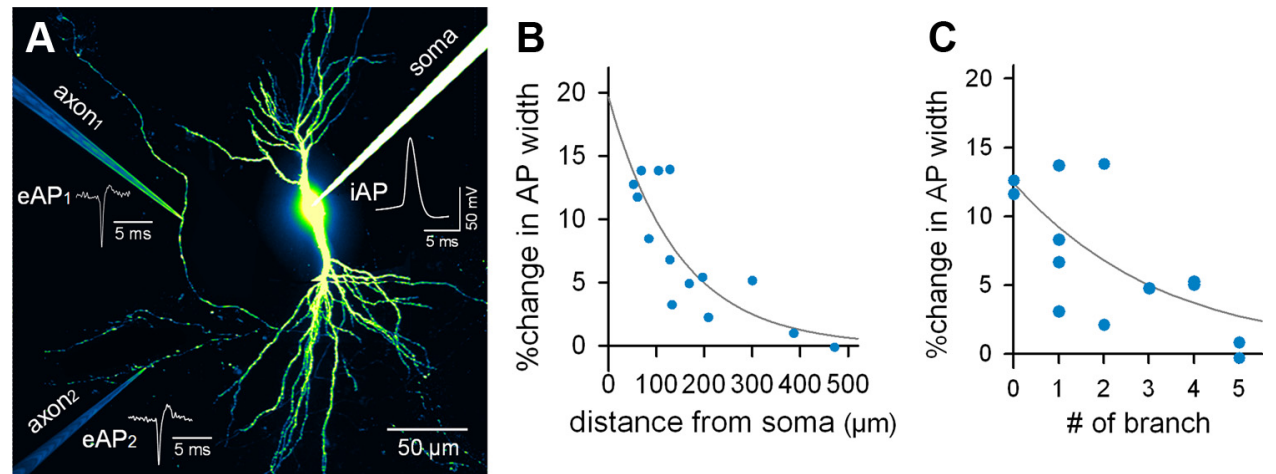

Figure 5. Depolarization-broadened APs return to a normal width along the length of the axon and through the branch points they traverse. $A$, Representative image of dual, cell-attached recordings of eAPs from different axonal branches. $\boldsymbol{B}$, Distance-dependent recovery of depolarization-broadened APs. The curve indicates the least-square best fit of the exponential decay function $\left(\Delta\right.$ width $=19.6 \times e^{-d / 145} ; d$, distance from the soma; $\left.F_{(1,12)}=229.8 ; p=0.002\right) . C$, Branch number-dependent recovery of depolarization-broadened APs. The curve indicates the best fit of the exponential decay function $\left(\Delta\right.$ width $=12.4 \times e^{-n / 2.9} ; n$, number of upstream branches; $\left.F_{(1,12)}=158.4 ; p=0.006\right)$. Data presented here were collected from 14 recordings.
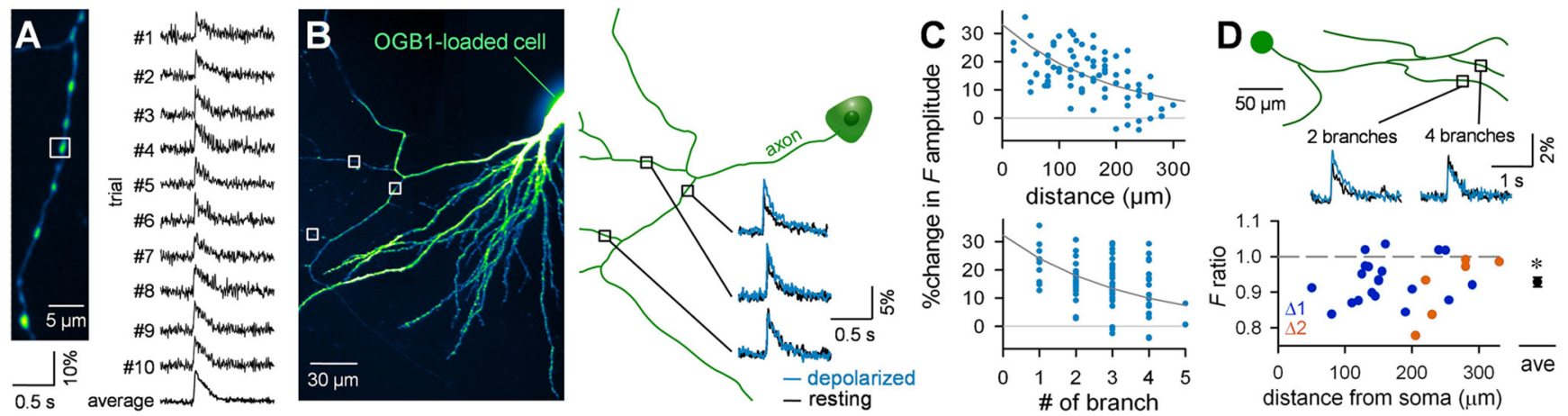

Figure 6. Depolarization-induced enhancementofAP-elicited calcium elevationsin presynapticterminalsisdependentontheaxonallengthandthenumber ofbranch pointsthroughwhichthe APs stravel. $A$, Nipkow-disk confocal calcium imaging from a single presynaptic terminal of a CA3 pyramidal cell. Ten successive traces and their average AP-induced calcium dynamics were obtained from the presynaptic terminal, indicated by the broken-linecircleintheleftphoto. Thebottom photowasthe $5 \times$ magnification of thewhileboxin the top photo. $\boldsymbol{B}$, Presynapticalciumimaging frommultipleaxon segments on asingle $C A 3$ pyramidal cell. The axontrajectory (right) was reconstructed from the confocal image taken at a low magnification (left). Traces indicate typical examples of AP-evoked calcium transients recorded from synaptic terminals in three axonal segments (boxed) of a resting (black) or $20 \mathrm{mV}$-depolarized CA3 neuron (blue).C, Top, Distance-dependentrecovery of depolarization-enhanced calcium responses. The curveindicates theleast-squares bestfitfor a single exponential decay function $\left(\% \Delta\right.$ amplitude $=33.1 \times e^{-d / 176} ; d$, distance from the soma; $\left.F_{(1,79)}=30.2 ; p<0.001\right)$. Bottom, Branch number-dependent recovery of depolarization-enhanced calcium responses. The curve indicates the best fit for a single exponential decay function $\left(\% \Delta\right.$ amplitude $=32.3 \times e^{-n / 3.4} ; n$, number of upstream branches; $\left.F_{(1,79)}=11.2 ; p=0.0012\right)$. Data presented here were collected from 81 axonal segments from seven cells. D, Top, Representative AP-evoked calcium transients in a resting (black) or $20 \mathrm{mV}$-depolarized neuron (blue) wererecorded from two axon segments ata distance of $209 \mu \mathrm{m}$ from the soma. Thenumbers of branch points from the somaweretwo (left) andfour (right). Bottom, Ratios of depolarization-induced enhancement of calcium transients atmorebranched axonal segments to that atless branched axonal segments are plotted against the distance from the soma. Colors indicate the difference in branching number between two axonal segments. ${ }^{*} p=2 \times 10^{-5}$ (paired t test; $n=28$ pairs from 13 cells).

7B). Somatic depolarization increased the close CA3-to-CA3 synaptic efficacy compared with the efficacy at the resting potential $\left(n=11\right.$ pairs; $t_{(10)}=2.40 ; p=0.04$, paired $t$ test), whereas no depolarization-induced facilitation occurred at distant CA3-to-CA3 $\left(n=13\right.$ pairs; $t_{(12)}=$ $2.01 ; p=0.11$, paired $t$ test $)$ or CA3-to-CA1 synapses $\left(n=12\right.$ pairs; $t_{(11)}=1.38 ; p=$ 0.19 , paired $t$ test).

The ROTing technique requires intracellular loading of calcium dyes into presynaptic neurons. Therefore, dye-mediated calcium chelating might interfere with calcium-dependent process associated with synaptic vesicle release and thereby affect our data. To exclude this possibility, we searched five close CA3-to-CA3 pairs and four distant CA3-to-CA3 pairs by random patching from two or three neurons, without the aid of ROTing. In these naive pairs, we obtained similar results (Fig. $7 B$; close
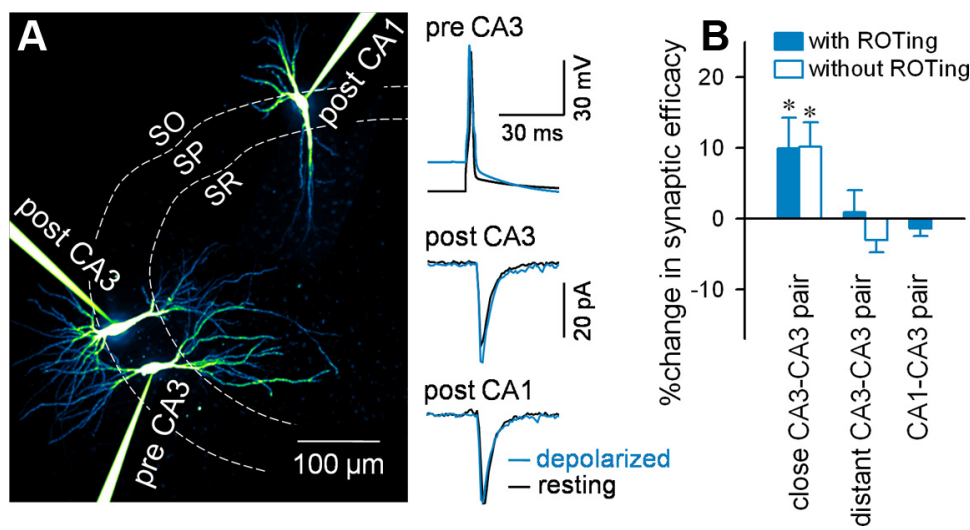

Figure 7. Somatic depolarization of CA3 pyramidal cells failitates CA3-to-CA3 but not CA3-to-CA1 synaptic transmission. $A$, Representative traces of triple recordings from a presynaptic CA3 pyramidal cell and postsynaptic CA3 (close target) and CA1 (distant target) pyramidal cells. Right traces indicate uEPSCS at CA3-to-CA3 (center) and CA3-to-CA1 synapses (bottom) in response to single APs in a presynaptic CA3 neuron (top) in a resting (black) or $20 \mathrm{mV}$ depolarized state (blue). Fifty trials were averaged for each trace. $B$, Summary graph depicting somatic depolarizationinduced changes in synaptic efficacy between CA3-to-CA3 neuron pairslocated within $100 \mu \mathrm{m}$ (doseCA3-CA3 pair), CA3-to-CA3 pairs separated by $>300 \mu \mathrm{m}$ (distant CA3-CA3 pair), and CA3-to-CA1 pairs (CA1-CA3 pair). CA3-CA3 neuron pairs were searched with (closed column) and without ROTing (open column). Using ROTing, data were collectedfrom 11 doseCA3-CA3 pairs, 13 distantCA3-CA3 pairs, and 12CA3-CA1 pairs ( ${ }^{*} p=0.04$, paired $t$ test). Without ROTing, data were collected from five close and four distant $C A 3-C A 3$ pairs $\left({ }^{*} p=0.03\right.$, paired $t$ test). 

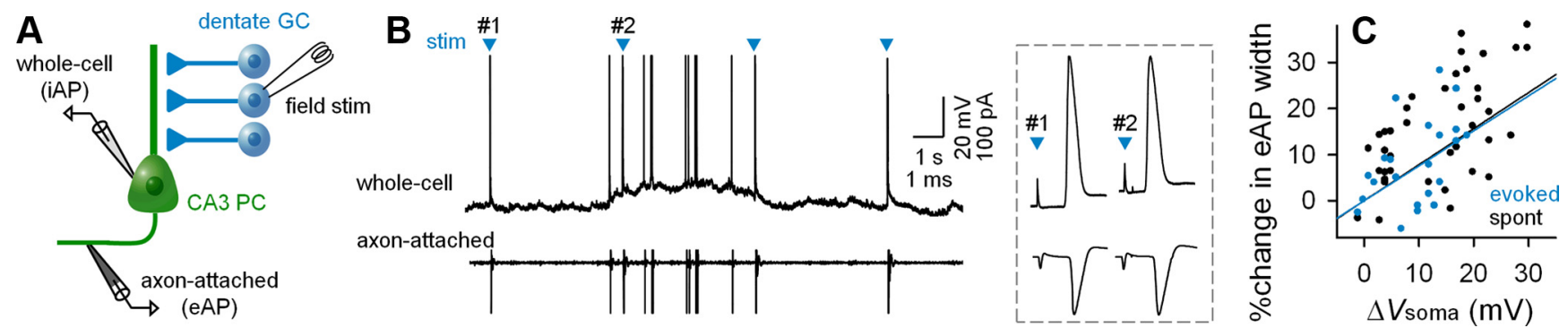

Figure 8. Spontaneous depolarization results in AP broadening. $A$, Experimental design. The granule cell (GC) layer of the dentate gyrus was stimulated, and the membrane potential and iAPs were whole-cell recorded from a CA3 pyramidal cell (PC). eAPs were recorded from the axon in the cell-attached mode. Intrinsic neural activity was enhanced by low divalent cation concentrations. $B$, Representative whole-cell (top) and cell-attached (bottom) traces. Blue arrowheads indicate the times of electric stimulation. APs indicated by \#1 (resting) and \#2 (depolarized) are magnified $1000 \times$ in the inset. C, AP broadening was positively correlated with membrane potential at the time of AP initiation. This correlation was observed in stimulation-evoked (blue; $r=0.53, t_{(24)}=$ $3.04, p=0.006)$ and spontaneous APs (black; $r=0.48, t_{(29)}=4.36, p<0.0001$ ).

CA3-to-CA3, $t_{(4)}=3.26, p=0.03$, paired $t$ test; distant CA3 -toCA3, $n=4$ pairs, $t_{(3)}=2.11, p=0.12$, paired $t$ test).

\section{AP-width modulation by spontaneous network activity}

Finally, to determine whether the AP width is subject to modulation by intrinsic fluctuations of subthreshold $\Delta V_{\text {soma }}$, we modified the ionic composition of the ACSF to enhance the level of spontaneous activity (Sanchez-Vives and McCormick, 2000; Tsukamoto-Yasui et al., 2007; Lu et al., 2010). Somatic membrane potentials and axonal eAPs were simultaneously recorded from a CA3 pyramidal neuron. Hippocampal mossy fibers were stimulated by electrodes placed on the dentate hilus to mimic synchronous synaptic inputs from dentate granule cells (Fig. $8 A$ ). CA3 pyramidal neurons exhibited spontaneous fluctuations in subthreshold membrane potentials with spontaneous or stimulievoked APs (Fig. 8B). Changes in axonal eAP width correlated positively with the voltage differences between the resting potential and the mean membrane potentials $1-50 \mathrm{~ms}$ before the APs (Fig. 8C). This positive relationship was observed in both evoked $\left(r=0.53 ; t_{(24)}=3.04 ; p=0.006\right)$ and spontaneous APs $(r=0.48$; $\left.t_{(29)}=4.36 ; p<0.0001\right)$. Our HH model simulation predicted that this relationship should be exponential rather than linear. Exact resting potentials could not be determined in the experiments, however, due to continuous, incoming synaptic activity. Thus, $\Delta V_{\text {soma }}$ was calculated as a voltage difference from the average of the minimal $1-10 \%$ values of membrane potentials during the observed period (putative resting potential), and the relationship to the AP width was fitted to a simple linear function. Regardless of whether the relationship is linear or nonlinear, our data indicate that, similar to artificially induced $\Delta V_{\text {soma, }}$, intrinsically occurring depolarization produces AP broadening, suggesting a physiological relevance for the impact of the internal state of a neuron on information transfer.

\section{Discussion}

In the present study, we demonstrated that $\Delta V_{\text {soma-induced }}$ modulation of the AP waveform and presynaptic calcium dynamics decayed depending on the length of the axon and the number of branch points over which AP passed. Unfortunately, the dependence on the number of branch points was not quantitatively shown by our experimental data, but it was confirmed by our $\mathrm{HH}$ model and was consistent with the calcium-imaging data. Based on our anatomical and computational analyses, we consider that this branch-dependent recovery of the distorted APs is at least in part due to branch-dependent narrowing of axonal fibers. However, we do not rule out the involvement of other unknown factors. In addition, the facilitatory effect of
$\Delta V_{\text {soma }}$ on synaptic transmission was observed at nearby but not distant synapses.

In neocortical pyramidal cells and dentate granule cells in acute slice preparations, it has been shown that axonal depolarization by somatic depolarization is reduced with a length constant $\lambda$ of 400-550 $\mu \mathrm{m}$ (Alle and Geiger, 2006; Shu et al., 2006; Kole et al., 2007). The present work proposes that this $\lambda$ value may overestimate the true range of the $\Delta V_{\text {soma }}$ influence in some types of neurons because of to two facts: (1) $\lambda_{\mathrm{AP}}$ is smaller than $\lambda$, and (2) the axonal diameter is spatially inhomogeneous.

A nonlinear relationship between $\lambda_{\mathrm{AP}}$ and $\lambda$ was also reported in layer 5 neocortical neurons (Kole et al., 2007). This is a natural consequence of the nonlinear relationship between $\Delta V_{\text {soma }}$ and the AP width; depolarization increased the AP width with a rate varying depending on the degree of depolarization. Our pharmacological investigations indicate that the waveform modulation of an AP occurs at the late phase of the AP and is probably caused by a change in the kinetics of voltage-sensitive potassium channels. The availability of potassium channels is well known to depend on the membrane potential at which an AP is initiated. Consistent with this, we succeeded in replicating the nonlinear relationship between $\Delta V_{\text {soma }}$ and the AP width by incorporating a voltage-dependent inactivation curve of potassium conductance into the $\mathrm{HH}$ model; note that the conventional $\mathrm{HH}$ neuron generates a stereotyped $\mathrm{AP}$ and does not exhibit $\Delta V_{\text {soma }}$-induced AP broadening. Another important consequence of the nonlinear relationship between $\Delta V_{\text {soma }}$ and the AP width is that the relationship between $\lambda_{\mathrm{AP}}$ and $\lambda$ depends on $\Delta V_{\text {soma }} ; \lambda_{\mathrm{AP}}$ is smaller than $\lambda$ to more extents at larger $\Delta V_{\text {soma }}$. Therefore, the effect of $\Delta V_{\text {soma }}$ drops more rapidly along the axon length under more depolarized states.

We found that $\Delta V_{\text {soma }}$-augmented presynaptic calcium dynamics decayed with $\lambda_{\text {calcium }}=176 \mu \mathrm{m}$, which is similar to $\lambda_{\mathrm{AP}}$. Therefore, it is unlikely that the influence of $\Delta V_{\text {soma }}$ on synaptic transmission reaches the distance expected simply by $\lambda$. However, it remains controversial whether $\Delta V_{\text {soma }}$-induced synaptic facilitation is mediated by modification of the AP waveform or presynaptic calcium dynamics. In cortical principal cells, $\Delta V_{\text {soma }}$ modulates the AP waveform by changing Kv1-type potassium channel kinetics (Kole et al., 2007; Shu et al., 2007). In contrast, presynaptic depolarization at the calyx of Held facilitates synaptic transmission without distorting AP waveforms (Awatramani et al., 2005). $\Delta V_{\text {soma }}$-induced synaptic facilitation is mediated by calcium channel facilitation at the calyx of Held (Awatramani et al., 2005; Hori and Takahashi, 2009) and at GABAergic synapses in the molecular layer of the cerebellum (Christie et al., 2011) but 
not at hippocampal mossy fiber synapses (Alle and Geiger, 2006; Scott et al., 2008). Therefore, the mechanism underlying the $\Delta V_{\text {soma }}$ modulation of synaptic efficacy differs depending on the type of synapse. Our current study provides the first evidence that $\Delta V_{\text {soma }}$ affects both the AP waveform and the presynaptic calcium dynamics at recurrent fibers between hippocampal CA3 pyramidal cells.

Our imaging data must be interpreted with caution because we used a relatively high concentration $(250 \mu \mathrm{M})$ of OGB1, a high-affinity calcium probe, to monitor calcium levels in presynaptic terminals. Because the dyes used to monitor calcium dynamics in distant synapses had to diffuse intra-axonally over a long range, we could not achieve usable signal-to-noise ratios for OGB1 fluorescence at lower concentrations or with other lowaffinity calcium probes (Yu et al., 2010). The development of highly sensitive, genetically encoded calcium indicators will help clarify the true relationship between $\Delta V_{\text {soma }}$ and presynaptic calcium dynamics.

Although the decay constants $\left(\lambda, \lambda_{\mathrm{AP}}\right.$, and $\left.\lambda_{\text {calcium }}\right)$ may vary between experimental systems, the data are consistent and suggest that $\Delta V_{\text {soma }}$ influences local but not distant synapses. Indeed, we found that at associational recurrent fibers of hippocampal CA3 pyramidal cells, the facilitatory effect of somatic depolarization was observed only at close CA3-to-CA3 synapses, but not distant CA3-to-CA3 or CA3-to-CA1 synapses. This targetselective synaptic facilitation is likely due to the axon distance as well as branch points between the soma and the synapses recorded, because the axons of CA3 pyramidal cells are highly arborized even in the vicinity of the soma. Thus, the somatic membrane potential of these neurons modified the internal computational operation in the local circuit without affecting longprojection output. If this locally selective modulation is the case in the neocortex, such differential modulations may work to differentiate the modes of intracolumnar and intercolumnar communications. In any case, excitatory projection neurons in the neocortex and hippocampus typically form synaptic connections with both local and distant target neurons simultaneously. Therefore, it is plausible that these neurons differentially modulate proximal and distal synaptic outputs depending on the activity states of the local network, e.g., active (depolarized, UP) or quiescent (hyperpolarized, DOWN) states.

\section{References}

Alle H, Geiger JR (2006) Combined analog and action potential coding in hippocampal mossy fibers. Science 311:1290-1293.

Alle H, Geiger JR (2008) Analog signalling in mammalian cortical axons. Curr Opin Neurobiol 18:314-320.

Awatramani GB, Price GD, Trussell LO (2005) Modulation of transmitter release by presynaptic resting potential and background calcium levels. Neuron 48:109-121.

Christie JM, Chiu DN, Jahr CE (2011) $\mathrm{Ca}^{2+}$-dependent enhancement of release by subthreshold somatic depolarization. Nat Neurosci 14:62-68.

Conchello JA, Lichtman JW (2005) Optical sectioning microscopy. Nat Methods 2:920-931.

Dayan P, Abbott LF (2001) Theoretical neuroscience: computational and mathematical modeling of neural systems. Cambridge, MA: Massachusetts Institute of Technology.

Desmond NL, Levy WB (1984) Dendritic caliber and the 3/2 power relationship of dentate granule cells. J Comp Neurol 227:589-596.

Hodgkin AL, Huxley AF (1952) A quantitative description of membrane current and its application to conduction and excitation in nerve. J Physiol 117:500-544.
Hori T, Takahashi T (2009) Mechanisms underlying short-term modulation of transmitter release by presynaptic depolarization. J Physiol 587:2987-3000.

Ishikawa D, Takahashi N, Sasaki T, Usami A, Matsuki N, Ikegaya Y (2010) Fluorescent pipettes for optically targeted patch-clamp recordings. Neural Netw 23:669-672.

Khaliq ZM, Raman IM (2006) Relative contributions of axonal and somatic $\mathrm{Na}$ channels to action potential initiation in cerebellar Purkinje neurons. J Neurosci 26:1935-1944.

Kole MH, Letzkus JJ, Stuart GJ (2007) Axon initial segment Kv1 channels control axonal action potential waveform and synaptic efficacy. Neuron 55:633-647.

Koyama R, Muramatsu R, Sasaki T, Kimura R, Ueyama C, Tamura M, Tamura N, Ichikawa J, Takahashi N, Usami A, Yamada MK, Matsuki N, Ikegaya Y (2007) A low-cost method for brain slice cultures. J Pharmacol Sci 104:191-194.

Li XG, Somogyi P, Ylinen A, Buzsaki G (1994) The hippocampal CA3 network: an in vivo intracellular labeling study. J Comp Neurol 339:181-208.

Lu B, Zhang Q, Wang H, Wang Y, Nakayama M, Ren D (2010) Extracellular calcium controls background current and neuronal excitability via an UNC79-UNC80-NALCN cation channel complex. Neuron 68:488-499.

Perkins KL (2006) Cell-attached voltage-clamp and current-clamp recording and stimulation techniques in brain slices. J Neurosci Methods 154:1-18.

Raastad M, Shepherd GM (2003) Single-axon action potentials in the rat hippocampal cortex. J Physiol 548:745-752.

Rall W (1959) Branching dendritic trees and motoneuron membrane resistivity. Exp Neurol 1:491-527.

Sanchez-Vives MV, McCormick DA (2000) Cellular and network mechanisms of rhythmic recurrent activity in neocortex. Nat Neurosci 3:1027-1034.

Sasaki T, Matsuki N, Ikegaya Y (2007) Metastability of active CA3 networks. J Neurosci 27:517-528.

Sasaki T, Takahashi N, Matsuki N, Ikegaya Y (2008) Fast and accurate detection of action potentials from somatic calcium fluctuations. J Neurophysiol 100:1668-1676.

Sasaki T, Minamisawa G, Takahashi N, Matsuki N, Ikegaya Y (2009) Reverse optical trawling for synaptic connections in situ. J Neurophysiol 102:636-643.

Sasaki T, Matsuki N, Ikegaya Y (2011) Action-potential modulation during axonal conduction. Science 331:599-601.

Scott R, Ruiz A, Henneberger C, Kullmann DM, Rusakov DA (2008) Analog modulation of mossy fiber transmission is uncoupled from changes in presynaptic $\mathrm{Ca}^{2+}$. J Neurosci 28:7765-7773.

Shu Y, Hasenstaub A, Duque A, Yu Y, McCormick DA (2006) Modulation of intracortical synaptic potentials by presynaptic somatic membrane potential. Nature 441:761-765.

Shu Y, Yu Y, Yang J, McCormick DA (2007) Selective control of cortical axonal spikes by a slowly inactivating $\mathrm{K}^{+}$current. Proc Natl Acad Sci U S A 104:11453-11458.

Takahashi N, Sasaki T, Matsumoto W, Matsuki N, Ikegaya Y (2010) Circuit topology for synchronizing neurons in spontaneously active networks. Proc Natl Acad Sci U S A 107:10244-10249.

Takahashi N, Kitamura K, Matsuo N, Mayford M, Kano M, Matsuki N, Ikegaya Y (2012) Locally synchronized synaptic inputs. Science, in press.

Tsukamoto-Yasui M, Sasaki T, Matsumoto W, Hasegawa A, Toyoda T, Usami A, Kubota Y, Ochiai T, Hori T, Matsuki N, Ikegaya Y (2007) Active hippocampal networks undergo spontaneous synaptic modification. PLoS One 2:e1250.

Turner DA, Schwartzkroin PA (1980) Steady-state electrotonic analysis of intracellularly stained hippocampal neurons. J Neurophysiol 44:184-199.

Wittner L, Henze DA, Zaborszky L, Buzsaki G (2007) Three-dimensional reconstruction of the axon arbor of a CA3 pyramidal cell recorded and filled in vivo. Brain Struct Funct 212:75-83.

Yu Y, Maureira C, Liu X, McCormick D (2010) P/Q and N channels control baseline and spike-triggered calcium levels in neocortical axons and synaptic boutons. J Neurosci 30:11858-11869. 